\title{
MEDICIÓN DE ESTRÉS TÉRMICO EN LOS AMBIENTES DE UNA FÁBRICA DE CHOCOLATES EN LA CIUDAD DE LIMA
}

\author{
HEAT STRESS MEASUREMENT IN CHOCOLATE FACTORY ENVIRONMENTS \\ IN THE CITY OF LIMA
}

${ }^{1}$ Luis Yoza, ${ }^{2}$ Oscar Tang, ${ }^{3}$ Roxani Yaringaño \& ${ }^{4}$ Yolanda Huachaca

\begin{abstract}
Resumen
El presente trabajo tuvo como objetivo evaluar los niveles de estrés térmico en una planta de fabricación de chocolate ubicada en la ciudad de Lima, usando el método denominado Índice TBGH de acuerdo a la norma RM 375 -2008 NTP evaluándose paralelamente el gasto metabólico de cada trabajador. La evaluación fue efectuada en 2 etapas del proceso de fabricación en caliente denominados derretidora de licor y chocomill: El equipamiento usado consto de termómetros ambientales, termistores, equipo de medición de temperatura de globo y un equipo de medición de estres termico marca EXTECH. Los resultados obtenidos con el equipo EXTECH mostraron valores similares comparados con los medidos con los termómetros. Sin embargo, los medidos usando termistores dieron valores discrepantes con los primeros. Los resultados de las mediciones nos indican que los valores de TGBHi en las dos etapas del proceso están por debajo a lo establecido por la norma nacional.
\end{abstract}

Palabras clave: estrés térmico, gasto metabólico, temperatura de globo, índice TGBH.

\begin{abstract}
The present study aimed to evaluate the thermal stress levels in the chocolate manufacturing plant located in the city of Lima, using a method called TBGH index according to standard of the RM 375-2008 NTP. The evaluation was carried out in 2 stages of the manufacturing process called melted liquor and chocomill: The equipment used were environmental thermometers, thermistors, globe temperature equipment and measuring equipment EXTECH brand for thermal stress. The results with thermometers and equipment EXTECH showed similar values compared with those measured with themistors that gave discrepant values with the first. The main conclusion was that the measurements indicated that the WBGT values in the two stages of the process are below the national standard.

In analyzing the results of the estimates of the models that explain the daily volatility of the Profitability of General Index of the Lima Stock Exchange (RIGBVL) period 2009 - 2011 it was concluded that the GARCH $(1,1)$ model is the appropriate, because unlike the other models have the lowest value in both the Akaike information criterion (AIC) and the Schwarz information criterion (BIC). Previously RIGBVL mean the AR model (1) was modeled.
\end{abstract}

Keys words: Heat stress, metabolic rate, globe temperature, WBGT index.

\section{Introducción}

El rápido desarrollo industrial de los últimos años en el país ha venido acompañado de las quejas de los trabajadores a las condiciones ambientales dentro del trabajo en especial las relacionadas a las altas temperaturas del aire, fuentes de calor radiante, alta humedad y contacto con fuentes calientes, que en algunas con ambientes cerrados como las industrias de fundición, plantas de cerámicas, fabricas de vidrio, plásticos, panaderías, industrias y la industria minera; y en otras en ambientes externos a cielo abierto como la construcción, reciclaje en climas cálidos, afectan la salud y la eficiencia de los trabajadores. En los últimos años en el Perú se han venido dando normas que permiten regular las exposiciones a las altas temperaturas como la Norma Básica de Ergonomía y de Procedimiento de Riesgo Disergonómico (RM- 75-2008-TR); Reglamento de Seguridad y Salud Ocupacional y otras medidas complementarias en Minería (DS 055-2010-MEM -2010- guía 2; y la Seguridad Industrial (DS 42F). y al norma internacional ISO 7243-1982 Hot EnvironmentsEstimation of the heat stress on working man based on WBGT index (wet bulb and globe temperature)

El objetivo principal del presente trabajo es evaluar el nivel de estrés térmico en dos procesos en caliente en la industria de chocolate como son, los de molienda (chocomill) y los de derretido del chocolate (derretidora de licor), en una planta ubicada en la ciudad de Lima. Para lo cual, se ha utilizado el método de índice de

\footnotetext{
1,2,3Universidad Nacional Agraria la Molina, Lima, Perú. E-mail: otang@lamolina.edu.pe

${ }^{4}$ Asesora en seguridad Industrial. Lima, Perú.
} 
temperatura bulbo seco, bulbo húmedo y temperatura de globo (Índice TBGH) ver Iñaki A (sin fecha). De otro lado, se ha considerado un segundo objetivo al comparar los valores encontrados con las mediciones directas con termómetros y termistores con los encontrados con el equipo EXTECH TH30. Los valores del índice TBGH se medirán usando termómetros de columna de mercurio y termistores para las temperaturas de bulbo húmedo; y para las temperaturas de globo por medio de la esfera de cobre pintada de negro.

\section{Materiales y métodos}

Los gastos metabólicos fueron estimados de acuerdo a las distintas actividades efectuadas por los trabajadores y en base las normas existentes y el tipo de categoría de trabajo es de acuerdo a la ACGIH (2007). tabla 1.

Tabla 1. Categorías de gastos metabólicos para una persona de $70 \mathrm{~kg}$.

\begin{tabular}{lcc}
\hline Categoría & $\begin{array}{c}\text { Gastos metabólicos } \\
(\mathrm{W}) \text { promedio }\end{array}$ & $\begin{array}{c}\text { Rango metabólico } \\
(\mathrm{W})\end{array}$ \\
\hline Descanso & 115 & --- \\
Ligero & 180 & $115-235$ \\
Moderado & 300 & $235-360$ \\
Pesado & 415 & $360-470$ \\
Muy Pesado & 520 & ----- \\
\hline
\end{tabular}

Fuente ACGIH (2007)

Para la evaluación del Estrés Térmico en la planta se usará el método TGBHi Temperatura de bulbo húmedo y temperatura de globo en ambientes interiores de acuerdo a la norma RM 375 -2008, y la ecuación:

$T G B H i=T B H x 0.7+T G x 0.3$

Las mediciones de los valores de TGBHi serán obtenidos de 3 formas: Con las mediciones por medio de termómetros, termistores, y con el equipo EXTECH TH30. Los cuales serán contrastados usando un termómetro R. Fues Berlin Steglitz de $\pm 0.25^{\circ} \mathrm{C}$ de precisión del Observatorio Meteorológico Von Humboldt de la Universidad Nacional Agraria La Molina.
Los valores limites máximos permisibles de comparación están dadas por la norma RM 375-2008 y a las recomendaciones de la ACGIH 2007; como se muestra en la tabla 2.

Aclimatización: Es un proceso de adaptación fisiológica que incrementa la tolerancia a ambientes calurosos, fundamentalmente por variación del flujo de sudor y del ritmo cardíaco. La aclimatación es un proceso necesario, que debe realizarse a lo largo de 6 o 7 días de trabajo, incrementando poco a poco la exposición al calor. Se considera que un trabajador está aclimatizado si ha realizado un trabajo con exposición a calor en al menos 5 de los últimos 7 días o 10 de los últimos 14 días. Sin embargo, los beneficios de la aclimatización se pierden fácilmente si las variaciones en la temperatura son importantes (elevaciones repentinas) o si no ha habido exposición en más de 4 días.

Límite Máximo Permisible: El LMP representa la condición en el cual el trabajador se encuentra en condiciones de aclimatación, adecuadamente hidratado, sano y no medicado; y que puede ser expuesto repetidamente a las mismas condiciones sin efectos adversos a su salud. ACGIH (2007). Es el valor máximo que debe alcanzar la temperatura TGBH en un ambiente caluroso. Sin embargo, no es una frontera definida entre condiciones seguras e inseguras, por lo que se recomienda siempre usarlo junto con el Nivel de Acción.

Nivel de Acción: Valor de temperatura TGBH por encima del cual se deberá empezar a tomar medidas correctivas y preventivas para hacer frente a la exposición a calor en el ambiente de trabajo. Además, se deberá adoptar medidas de vigilancia médica que garanticen la no presencia de síntomas y signos del estrés térmico. El nivel de acción es equivalente a las condiciones de un trabajador no aclimatizado. ACGIH (2007)

De otro lado, cabe señalar la norma DS 0552010 en su Guía 2 donde se enfoca las condiciones de exposición en base a los LMP y al nivel de acción, de acuerdo con las recomendaciones de la ACGIH. Anexo 1.

Tabla 2. Valores límites máximos permisibles para el índice TGBH de acuerdo con la categoría de trabajo y el porcentaje de trabajo descanso. En ${ }^{\circ} \mathrm{C}$.

\begin{tabular}{|c|c|c|c|c|c|c|c|c|}
\hline \multirow{2}{*}{ Tipo de trabajo } & \multicolumn{4}{|c|}{ Aclimatado $\left({ }^{\circ} \mathrm{C}\right)$} & \multicolumn{4}{|c|}{ No Aclimatado $\left({ }^{\circ} \mathrm{C}\right)$} \\
\hline & Liviano & Moderado & Pesado & Muy pesado & Liviano & Moderado & Pesado & Muy pesado \\
\hline $\begin{array}{l}\text { 100\%Tra. } \\
0 \% \text { Des. }\end{array}$ & 29.5 & 27.5 & 26.0 & ----- & 27.5 & 25.0 & 22.5 & -- \\
\hline $\begin{array}{l}75 \% \text { Tra } \\
25 \% \text { des. }\end{array}$ & 30.5 & 28.5 & 27.8 & ------ & 29.0 & 26.5 & 24.5 & ---- \\
\hline $\begin{array}{l}50 \% \text { Tra. } \\
50 \% \text { Des. }\end{array}$ & 31.5 & 29.5 & 28.5 & 27.5 & 30.0 & 28.0 & 26.5 & 25.0 \\
\hline $\begin{array}{l}25 \% \text { Tra. } \\
75 \% \text { Des }\end{array}$ & 32.5 & 31.0 & 30. & 29.5 & 31.0 & 29.0 & 28.0 & 26.5 \\
\hline
\end{tabular}

Fuente:RM 375 -2008 TR 
Equipos. Los instrumentos usados fueron: termómetros de mercurio de $\pm 0.5^{\circ} \mathrm{C}$ de precisión, termistores de 250 Ohmios de escala con calibración al $\pm 0.5^{\circ} \mathrm{C}$ de precisión, esfera de cobre de $10 \mathrm{~cm}$ de diámetro pintada de negro para la medición de la temperatura de globo y un equipo de estrés térmico marca EXTECH. Modelo: TH30.

\section{Resultados y discusión}

Las mediciones efectuadas con el equipo EXTECH, termómetro y termistores fueron evaluados estadísticamente usando la diferencia de medias comprobándose que los medidos con el equipo EXTECH y los termómetros son significativamente similares, por lo que se procedió a desechar los obtenidos con los termistores, debido posiblemente a que la posición y el procedimiento de humedecer el termistor no fue adecuado.

En el tabla 3 se muestran los valores obtenidos en el área de CHOCOMILL, habiéndose medido las temperaturas a la altura de $1.1 \mathrm{~m}$ durante 1 hora desde las $11 \mathrm{am}$. hasta las 12am. en intervalos de 15 minutos cada uno durante el día 08 de agosto del 2013, La Figura 1 muestra el proceso de medición en el área de Chocomill.

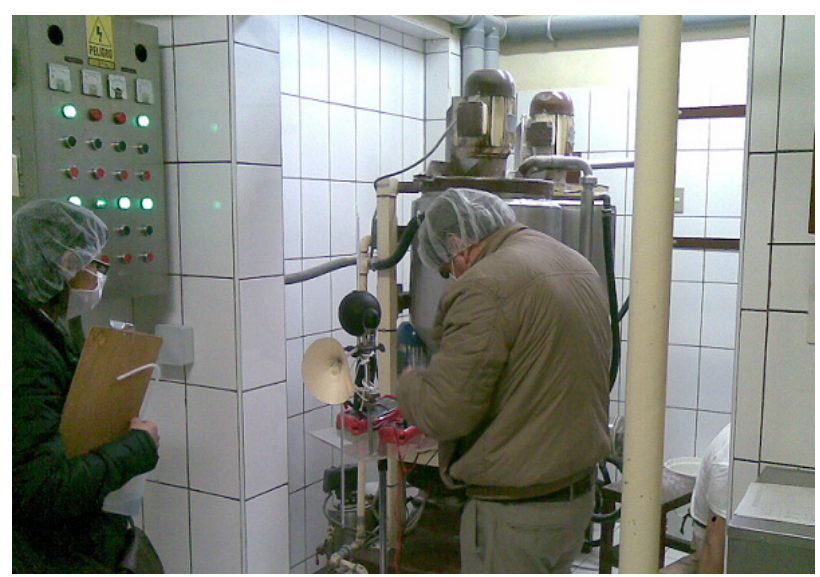

Figura 1. Foto de la medicion en el area de Chocomill.

Los valores mostrados del Indice TGBHi mostrados en las tablas 3 y 4 fueron calculados con la relación 1, para mediciones de estrés térmico en interiores.

Tabla 3. Datos de temperatura y TGBHi del área de CHOCOMIll para el equipo EXTECH H30 y termómetro.

\begin{tabular}{|c|c|c|c|c|c|c|c|c|}
\hline & \multicolumn{4}{|c|}{ Equipo EXTECH } & \multicolumn{4}{|c|}{ Termómetro } \\
\hline & $\begin{array}{l}\text { Temp. } \\
\text { B.Seco } \\
\left({ }^{\circ} \mathrm{C}\right)\end{array}$ & $\begin{array}{c}\text { Temp. } \\
\text { Globo }\left({ }^{\circ} \mathrm{C}\right)\end{array}$ & $\begin{array}{l}\text { TGBHi } \\
\left({ }^{\circ} \mathrm{C}\right)\end{array}$ & $\begin{array}{l}\text { Humedad } \\
(\%)\end{array}$ & $\begin{array}{l}\text { Temp. } \\
\text { B.seco } \\
\left({ }^{\circ} \mathrm{C}\right)\end{array}$ & $\begin{array}{c}\text { Temp. B. } \\
\text { Humedo } \\
(\%)\end{array}$ & $\begin{array}{c}\text { Temp. } \\
\text { Globo }\left({ }^{\circ} \mathrm{C}\right)\end{array}$ & $\begin{array}{c}\text { TGBHi } \\
\left({ }^{\circ} \mathrm{C}\right)\end{array}$ \\
\hline Media & 29.3 & 29.9 & 23.1 & 43.8 & 29.2 & 21.1 & 29.2 & 23.6 \\
\hline DS. & \pm 0.2 & \pm 0.4 & \pm 0.2 & \pm 1.3 & \pm 0.7 & \pm 0.6 & \pm 0.8 & \pm 0.5 \\
\hline CV $(\%)$ & 0.7 & 1.2 & 0.9 & 0.5 & 0.7 & 0.9 & 2.7 & 0.8 \\
\hline
\end{tabular}

En el tabla 4 se muestran los valores obtenidos en el área Derretidora de Licor, habiéndose medido las temperaturas a la altura de $1.1 \mathrm{~m}$, desde las $12.30 \mathrm{am}$. hasta las $14.45 \mathrm{am}$.

Tabla 4. Datos de de temperatura y TGBHi del área de DERRETIDORA DE LICOR para el equipo EXTECH H30 y termómetro.

\begin{tabular}{ccccccccc}
\cline { 2 - 9 } & \multicolumn{4}{c}{ Equipo EXTECH } & \multicolumn{4}{c}{ Termómetro } \\
\cline { 2 - 9 } & Temp. & $\begin{array}{c}\text { Temp. } \\
\text { B.Seco }\left({ }^{\circ} \mathrm{C}\right)\end{array}$ & $\begin{array}{c}\text { TGBHi } \\
\text { Globo }\left({ }^{\circ} \mathrm{C}\right)\end{array}$ & $\begin{array}{c}\text { Humedad } \\
(\%)\end{array}$ & $\begin{array}{c}\text { Temp. } \\
\text { B.seco } \\
\left({ }^{\circ} \mathrm{C}\right)\end{array}$ & $\begin{array}{c}\text { Temp. B. } \\
\text { Humedo } \\
(\%)\end{array}$ & $\begin{array}{c}\text { Temp. Globo }\left({ }^{\circ} \mathrm{C}\right) \\
\text { GGBHi } \\
\left({ }^{\circ} \mathrm{C}\right)\end{array}$ \\
\hline Media & 31.7 & 32.7 & 24.8 & 40.4 & 31.5 & 23.4 & 32.5 & 26.2 \\
DS. & \pm 0.4 & \pm 0.2 & \pm 0.3 & \pm 1.7 & \pm 0.5 & \pm 0.5 & \pm 0.2 & \pm 0.4 \\
CV $(\%)$ & 1.1 & 0.6 & 1.0 & 4.2 & 1.6 & 1.9 & 0.6 & 1.4 \\
\hline
\end{tabular}

En la tabla 5 se muestran los valores promedios de las temperaturas de bulbo húmedo, globo y los valores del índice TGBHi obtenidos por el equipo EXTECH y por los termómetros. A los valores encontrados, como ya se indicó, se les aplico la prueba de diferencia de medias para determinar su similitud; obteniéndose para la temperatura de globo de la esfera negra de $10 \mathrm{~cm}$ y la del equipo EXTECH la prueba de $\mathrm{F}=1.28$ con la probabilidad de $\mathrm{p} \leq 0.29$ nos permite tomar como hipótesis nula que la variables no son compatibles debido a que $\mathrm{p}>0.05$ y con la prueba $\mathrm{t}$ con valores de $\mathrm{t}(-1.8) \mathrm{p} \leq$ 0.105 ; con la probabidad mayor que 0.05 lo que muestra que las medidas si son compatibles. De la misma forma, para el índice TGBHi, se comparo los valores obtenidos con las mediciones efectuadas con el globo de $10 \mathrm{~cm}$ y la temperatura de bulbo húmedo (ecuación 1) y las medidas con el equipo EXTECH; dando para la prueba $\mathrm{F}=2.34$ y la probabilidad de $\mathrm{p} \leq 0.165$ lo que permite tomar como hipótesis nula como no compatibles, con la prueba tigual a $t(1.7)$ y $p \leq 0.119$ mayor que 0.05 , valida la hipótesis de compatibilidad entre las variables.

La tabla 5, también muestra los valores del gasto metabólico de los trabajadores en las actividades 
estudiadas estimándose para ambos caso valores que oscilan entre 167 y $200 \mathrm{~W} / \mathrm{m}^{2}$ y que corresponde a la categoría actividad ligera para ambas actividades y además considerándose aceptable la razón trabajo/ descanso en $75 \% / 25 \%$ en condición de aclimatado ver tabla 2. Estando los valores encontrados están sujetos a la Norma Técnica Peruana (NTP) RM 375 -2008.

Tabla 5. Promedio de valores medidos y calculados usando el equipo EXTECH y termómetros para la razón trabajo/ descanso $75 \% / 25 \%$.

\begin{tabular}{ccccccc}
\hline \multirow{2}{*}{ Actividad } & & $\begin{array}{c}\text { Temp. Bulbo } \\
\text { Húmedo }\left({ }^{\circ} \mathrm{C}\right)\end{array}$ & $\begin{array}{c}\text { Temp. Globo } \\
\left({ }^{\circ} \mathrm{C}\right)\end{array}$ & $\begin{array}{c}\text { Gasto metabólico } \\
(\mathrm{W} / \mathrm{m} 2)\end{array}$ & $\begin{array}{c}\text { TGBHi } \\
\left({ }^{\circ} \mathrm{C}\right)\end{array}$ & $\begin{array}{c}\text { LMP TGBHi } \\
\left({ }^{\circ} \mathrm{C}\right)\end{array}$ \\
\hline \multirow{3}{*}{ Derretidora de licor } & Media & 23.4 & 32.6 & & 25.5 & \\
& $\mathrm{DS}$ & \pm 0.5 & \pm 0.2 & $167-200$ & \pm 0.8 & 30.5 \\
& $\mathrm{CV}(\%)$ & 2.2 & 0.6 & & 3.1 & 23.3 \\
Chocomill & Media & 21.1 & 29.6 & & & \\
& $\mathrm{DS}$ & \pm 0.6 & \pm 0.7 & $167-200$ & \pm 0.43 & 30.5 \\
& $\mathrm{CV}(\%)$ & 2.6 & 2.3 & & 1.8 & \\
\hline
\end{tabular}

En la tabla 5 se muestra los valores promediados de TGHi y comparados con la norma RM 375-2008 condición aclimatado; para la razón de trabajo/descanso de $75 \% / 25 \%$, encontrándose valor de $5.0^{\circ} \mathrm{C}$ y $7.2^{\circ} \mathrm{C}$ menores que el límite máximo permisible (LMP) para las tareas de la Derretidora de Licor y de Chocomill respectivamente.

\section{Conclusiones}

Los índices de TGBHi oscilan entre 23.3 y $25.5{ }^{\circ} \mathrm{C}$ para las zonas Chocolatemill y Derretidora de Licor respectivamente, encontrarse los mismos por debajo de los niveles del limite máximo permisible en 5.0 y $7.2{ }^{\circ} \mathrm{C}$ respectivamente, para la razón trabajo/descanso de $75 \% / 25 \%$, para trabajador con consumo metabólico ligero y aclimatado en ambos casos.

Los valores del gasto metabólico para las actividades evaluadas fluctúan entre $167-200 \mathrm{~W} / \mathrm{m}^{2}$ valores que corresponden a la actividad de nivel ligero aclimatado.

De otro lado, los valores encontrados con el equipo EXTECH H30 y los obtenidos con los termómetros como los medidos con el globo son compatibles, mostrándose que el equipo EXTECH puede ser usado con coeficiente de variación hasta en $3.1 \%$.

\section{Literatura citada}

ACGIH (American Conference of Governmental Industrial Hygienists), 2001. Documentation of the Threshold Limit Values for Physical Agents. $7^{\text {th }}$ Edition.

DS (Decreto Supremo) 055, 2010. Reglamento de Seguridad y Salud Ocupacional, Ministerio de Energía y Minas. PE.

DS ( Decreto Supremo) 42-F, 1964. Reglamento de Seguridad Industrial. PE

Iñaki, G. E. ( $\sin$ fecha). proximación AL Riesgo y Confort Térmico en el trabajo. ww.ergoKprevencion.org

NTP (Notas Técnicas de Prevención) 18, (sin fecha). Estrés Térmico Evaluación de las Exposiciones muy intensas, Instituto Nacional de Seguridad e Higiene en el Trabajo ES.

RM (Resolución Ministerial) 375, 2008. Norma Básica de Ergonomía y de Procedimiento de Riesgo Disergonómico. Ministerio de Trabajo y Promoción del Empleo. PE.

RM (Resolución Ministerial) 318, 2010. Modifican el Reglamento de Seguridad y Salud en el Trabajo de las Actividades Eléctricas, Ministerio de Energía y Minas. PE.

Finalmente se recomienda, efectuar evaluaciones en la época de verano donde los niveles de temperatura ambiental son mucho mayores.

Anexo 1. Norma DS 055, 2010 Guía 2 valores limites de TGBH. Valores límite de referencia para estrés térmico.

\begin{tabular}{ccccccccc}
\hline $\begin{array}{c}\text { Ubicación del trabajo } \\
\text { dentro de un ciclo } \\
\text { de trabajo- descanso }\end{array}$ & \multicolumn{3}{c}{ Valor Límite (TGBH en ${ }^{\circ} \mathrm{C}$ ) } & \multicolumn{3}{c}{ Nivel de Acción (TGBH en $\left.{ }^{\circ} \mathrm{C}\right)$} \\
\hline & Ligero & Moderado & Pesado & $\begin{array}{c}\text { Muy } \\
\text { Pesado }\end{array}$ & Ligero & Moderado & Pesado & $\begin{array}{c}\text { Muy } \\
\text { Pesado }\end{array}$ \\
\hline $75 \%$ a $100 \%$ & 31 & 28 & --- & --- & 28 & 25 & --- & --- \\
$50 \%$ a $75 \%$ & 31 & 29 & 27.5 & --- & 28.5 & 26 & 24 & --- \\
$25 \%$ a $50 \%$ & 32 & 30 & 29 & 28 & 29.5 & 27 & 25.5 & 24.5 \\
$0 \%$ a $25 \%$ & 32.5 & 31.5 & 30.5 & 30 & 30 & 29 & 28 & 27 \\
\hline
\end{tabular}

